Fecha de recepción: abril 2019

Fecha de aceptación: octubre 2019

Versión final: marzo 2020

\section{La evolución de los modelos de enseñar-aprender diseño en el nuevo escenario generacional}

Carmen Inés Galbusera Testa *

\begin{abstract}
Resumen: El enseñar-aprender diseño plantea desafíos en el contexto del paradigma global actual. Las generaciones de Baby-Boomers (1945-60) y X (1961-79), formadas en tiempos de guerra fría, son los organizadores didácticos de las generaciones NET o de nativos digitales: Millenials (1980-1993), Z (1994-2009) y Touch (2010-hoy). Los cambios cuantitativos y cualitativos se producen a velocidad acelerada en las dimensiones económicas, políticas, sociales, ambientales, tecnológicas y simbólicas desde fines del S.XX e inicios del XXI. Nuevas modalidades de enseñanza/aprendizaje producto de cambios generacionales, invitan a la reflexión crítica.
\end{abstract}

Palabras clave: enseñanza - aprendizaje - diseño - cambios - modelos - generaciones - reflexión crítica - contexto.

[Resúmenes en inglés y portugués en la página 114]

${ }^{(*)}$ Arquitecta Universidad de Belgrano 1980. Docente CBC-FADU-UBA 1985/actualidad. Docente Facultad Diseño y Comunicación Universidad de Palermo 2003/actualidad. Especialista en Docencia Universitaria FADU-UBA 2017. Especialista en Gestión Ambiental Metropolitana GAM-FADU-UBA 2015. Maestranda GAM-FADU-UBA. Investigadora PUC-FADU-UBA 2016/actualidad.

\title{
Introducción
}

¿Cuáles serían los principales cambios manifiestos en la enseñanza/aprendizaje del diseño en la educación superior? ¿Es posible identificar los modelos de aprendizaje que tienden a configurarse? ¿Qué impactos se perciben en los aprendizajes frente a la irrupción de las más recientes generaciones? ¿Cuáles son las generaciones que coexisten actualmente y cómo se facilitan las relaciones entre ellas? ¿Qué retos significativos presenta la Generación Net para la didáctica del SXXI? ¿Existen diferencias entre los propios estudiantes? Son muchos los interrogantes que estimulan la reflexión y el análisis. Los conceptos de enseñanza y aprendizaje del diseño, a la vez que el rol docente, deben ser redefinidos ante los nuevos escenarios del paradigma global actual.

La actividad de diseñar y su enseñanza, tiene una relación profunda y permanente con los procesos socio-culturales. Constituye un compromiso ético y de diálogo de los actores del 
diseño con la sociedad a la que pertenecen. De acuerdo a los avances obtenidos en algunos de los Encuentros de la Bienal Iberoamericana de Diseño (BID) realizados en Madrid en varias oportunidades, el objetivo principal de educar para y desde la innovación es ciertamente compatible con los valores más humanistas como la responsabilidad, el entusiasmo, la generosidad, la sinceridad, la empatía con el otro y con lo otro, el compromiso con el futuro y la pasión por diseñar "un mundo cada vez más abierto y justo". El diseñador es un actor clave e indispensable del cambio en las sociedades contemporáneas, independientemente del área o el contexto regional, nacional o internacional en que trabaja. El diseño implica comunidad y representa a la cultura como una construcción propia de una manera de habitar. El diseño actúa como gestor y generador de identidades, tanto en la escala local como en la global, en los espacios natural, social y económico. La actividad formativa del diseñador demanda del ejercicio usual, íntegro y valiente de la crítica tanto hacia su propio trabajo como hacia el medio social en el que se desenvuelve, como una garantía de ajuste y armonía constantes (5ºncuentro BID, 2013).

Los nuevos contenidos para la formación del diseñador deben ser transversales y transitivos: son procesos y habilidades orientados a capacitar al futuro profesional, superando la fragmentación del conocimiento transmitido en materias aisladas, relacionados con los contextos próximos, profundizando en estrategias y programas de acción para un autoaprendizaje continuo. Se propone formar a los futuros diseñadores con un espíritu emprendedor para que tengan iniciativa y sean capaces de generar sus propias propuestas liberando a los planes de estudio de rigideces impuestas por las instituciones de control, potenciando estrategias que favorezcan el aprendizaje significativo y se comporten como sistemas vivos, capaces de adaptarse y evolucionar de manera continua ( $5^{\circ}$ Encuentro BID, 2013).

Es tarea de todos los participantes en la enseñanza del diseño revalorizar su importancia en la sociedad, haciendo que éste sea entendido como agente de transformación y optimización permanente de la misma. Los centros formativos y de investigación en diseño pueden y deben actuar como laboratorio y espacio de generación del conocimiento, funcionando como agentes de cambio de los desafíos y problemas sociales que se viven. Una función inevitable de los centros debe ser también, acopiar, tramitar y propagar la memoria cultural de la disciplina del diseño. La comunidad del diseño y sus instituciones de formación se reconocen a sí mismas como comunidad de aprendizaje en la que todos, diseñadores, docentes, estudiantes, empresas, siguen aprendiendo constantemente y construyen su tarea a partir de la naturaleza, el ser humano y la historia (5º Encuentro BID, 2013).

\section{¿Cuáles serían los principales cambios manifiestos en la enseñanza/ aprendizaje del diseño?}

La formación en diseño necesita de instrumentos nuevos y actuales para implementar en las aulas y que la enseñanza del diseño necesita modelos de vínculo con las industrias, con la sociedad, con las ciudades y con la gente para hacer de la disciplina un segmento clave. En la actualidad, las instituciones afrontan el pasaje de los procesos formativos tradicionales hacia otros en los que las tecnologías tienen un peso específico importante; 
deberían asegurarse, a la vez, de que sus estudiantes alcancen las competencias necesarias para desenvolverse en medios laborales en los que el aprendizaje es incesante; deben competir, asimismo, en ámbitos internacionales y ser capaces de operar con la diversidad ( $6^{\circ}$ Encuentro BID, 2015).

La revolución digital y tecnológica coexisten con los vaivenes y la polarización social y cultural, a la vez que intervienen como factor de riqueza y acelerador de la dualidad, contribuyendo a desarrollar niveles nunca alcanzados de fortuna y prosperidad junto a patrones igualmente en aumento de precariedad y freno estructural. La tarea se enfrenta a nuevos retos, nuevos espacios y nuevos roles en donde se trabaja en equipos multidisciplinarios, interdisciplinarios y transversales y con sistemas abiertos y colaborativos. Esto implica una actualización de las maneras de relación y modelos de vinculación para dilucidar los marcos de referencia en los que se mueve el diseño, y una visión de las nuevas perspectivas que van del diseño perceptible al diseño intangible: diseño de servicios, diseño sostenible, diseño para la innovación social, diseño de información. El diseño visto como la articulación entre individuos y sus interacciones (7º Encuentro BID, 2017).

\section{¿Qué características tienen los modelos de aprendizaje que tienden a configurarse?}

Anna Escofet Roig, Iolanda Garcia Gonzalez y Begoña Gros Salvat desarrollan un profundo análisis y una clasificación basados en los nuevos y en los ya existentes modelos de aprendizaje observables actualmente en nuestras sociedades; los agrupan en tres categorías y tipos de aprendizaje: formal, no formal, informal.

En su investigación citan a Trinder, Guiller, Margaryan, Littlejohn y Nicol (2008, p. 13), quienes definen el aprendizaje formal como:

[...] el aprendizaje proporcionado por una institución educativa o de formación, estructurado (en términos de objetivos, temporalización y sistemas de apoyo al aprendizaje), implicando la presencia de un profesor o formador designado, $y$ dirigido a la obtención de una titulación, una certificación o acreditación.

Mientras que la segunda categoría, el aprendizaje informal, no supone ningún tipo de certificación. Es la consecuencia de las actividades sociales cotidianas relacionadas con la educación, el trabajo, la socialización con otros o la realización de actividades de ocio y hobbies. El aprendizaje informal puede ser estructurado o no estructurado, en términos de objetivos, tiempos y soporte del aprendizaje y puede ser intencional o no intencional (incidental) desde la perspectiva del aprendizaje (Escofet Roig, et al, 2011).

A su vez, apuntan las autoras, la Comisión Europea (European Commission, 2001) considera que una tercera categoría, el aprendizaje no formal, tiene lugar en un contexto institucional (e.g. un centro educativo o institución de formación), que es sistemático (en cuanto a la enunciación de propósitos de aprendizaje, duración del proceso y herramientas de enseñanza utilizadas), pero que no deriva en titulación o certificación oficiales reconocidas por el sistema educativo formal. Para el estudiante, el aprendizaje no formal es intencional 
y orientado a la obtención de unos objetivos determinados. Por el contrario, el aprendizaje informal tiene lugar en la cotidianeidad, en el trabajo, en la vida familiar o en el tiempo de ocio, pues no es estructurado ni organizado en los términos descriptos del formal y el no formal y corrientemente no implica ningún tipo de certificación.

Los autores Thomas y Seely Brown (2011), recurren a la expresión nuevas culturas de aprendizaje para mencionar aquellas formas que encarnan un concepto de raíz distinto de la creación y la distribución de conocimiento, y que confrontan claramente a la tradicional (Escofet Roig, et al, 2011).

\section{¿Qué impactos se perciben en los aprendizajes frente a la irrupción de las más recientes generaciones?}

En esta clasificación y descripción que proponen las autoras catalanas, se amplía que el aprendizaje informal aparenta constituir un subproducto o derivado de aprendizaje, que acontece espontáneamente y que, en general, no se considera claramente el objetivo o la consecuencia de ninguna maniobra. Además, la expresión comprende el conjunto de actividades de aprendizaje a desarrollar al margen de las tipologías establecidas de educación, con el objetivo intencionado de aprender, pero facilitadas en entornos informales. Este tipo de procesos -contrariamente a los formales y no formales- están organizados sólo por parte del propio sujeto que aprende. Los límites y relaciones entre ambos conceptos no son muy visiblemente abarcados y distinguibles. En la sociedad actual, las fronteras del aprendizaje formal e informal se desenfocan y problematizan. Este proceso se ha visto aumentado y evidenciado con el advenimiento de las tecnologías sociales (Escofet Roig et al, 2011).

Si bien Internet está presente e interviene en la vida diaria, hay que agregar el impacto social de la llamada web 2.0, expresión creada ad hoc para designar una nueva manera de concebir y usar la red, de intercambio social, que agrega un abanico de tecnologías a los usuarios de Internet y se actualiza continuamente: blogs, fórums, redes sociales, wikis, marcadores sociales y otras herramientas a través de las cuales compartir e intercambiar contenidos, crearlos en conjunto, rotularlos, etiquetarlos, exponerlos, re mezclarlos y re mixarlos, evaluarlos, etc. (Escofet Roig et al, 2011).

\section{¿Cuáles son las generaciones que coexisten actualmente y cómo se facilitan las relaciones entre ellas?}

La premisa fundamental para lograr un principio de entendimiento y comunicación entre diferentes generaciones es intentar conocer las características, las posibles raíces y condicionamientos de las mismas, con sus particularidades, considerando sus aspectos extraordinarios y sus limitaciones.

Varios autores utilizan la noción de generación como el conjunto de personas que comparten características distintivas según uno o varios criterios y que producen "comportamientos similares” en los miembros del grupo. En línea con esto según Ramón F. Ferrei- 
ro, el concepto de "generación" está vinculado a algún acontecimiento trascendente que produce transformaciones significativas en los integrantes de los grupos y de éste en su totalidad (Ferreiro R., 2000).

Un ejemplo clave de evento trascendente que afectó a los miembros de una generación, es de la Segunda Guerra Mundial, finalizada en 1945, que provocó en Estados Unidos de Norteamérica (EEUU) una explosión demográfica y cambios económicos, políticos y sociales que afectaron a los niños nacidos en el periodo posguerra. En coincidencia, el surgimiento de la televisión, por entonces la tecnología más poderosa en la historia, los marcaba a este grupo etario como generación y la sociedad cambiaba con la influencia de la misma. La ciencia de ese entonces le atribuyó el nombre de Generación Baby Boomer a todos aquellos que nacían, se educaban y desarrollaban en ese período (entre los años 1946 y 1964), ya que de manera evidente revelaban una forma de comportarse que lo definían como generación (Ferreiro R., 2000).

La publicación y éxito de una novela de Douglas Coupland, escritor canadiense que creó el término $X$ para describir a un grupo de personas nacidas y formadas desde mediados de la década de los sesenta del siglo XX en un contexto social y económico distinto al de sus padres y con perspectivas de vida también diferentes, ayudó a configurar el segundo ejemplo destacable: la Generación X. La abundancia económica de los años sesenta, los movimientos sociales de los países del Tercer Mundo, la guerra de Vietnam, entre otros sucesos, favorecen el surgimiento en EEUU y Canadá, según los investigadores de teoría de grupos, de una generación claramente reconocible: la Generación X (Ferreiro R., 2000). Las décadas de los ochenta y los noventa del siglo XX fueron escenario de la irrupción de las TIC, citadas anteriormente, cuestión que provocó cambios radicales y acelerados y llegó a plantear, más que una época de cambios, un cambio de época socio-cultural. En este contexto económico, social y cultural nace y se desarrolla una tercera generación intercedida por la evolución de las TIC y de la relación entre ambas: la telemática, concepto que refiere a la combinación de la informática y de la tecnología de la comunicación para el envío y la recepción de datos, responsable mayormente de un cambio de valoración de la realidad entre los miembros de este grupo de personas en pleno desarrollo, denominada como Generación Net, o N, o Net Gen, ya que de todas las TIC, computadora e internet constituyen sus insignias (Ferreiro R., 2000).

Nicolás Negroponte comenta en su libro "Ser Digital" que, como el acero en el siglo XIX y la electricidad en el XX, la revolución digital regulará la política, a economía y la sociedad del siglo XXI; se alterará la forma de aprender, de trabajar, de divertirse... toda la modalidad de vida. Lo anterior se alinea y refuerza con tendencias como la de la globalización o mundialización, como se sugiere decir en español, dado un aumento importante del comercio internacional y el intercambio cultural, la creciente comunicación e interdependencia entre los diferentes países del mundo (Ferreiro R., 2000).

El salto cualitativo se produce en la Net Gen al pasar de simples espectadores a usuarios con grandes posibilidades de participar, jugar, entretenerse, buscar, hacer, resolver, establecer relaciones, llevar a cabo proyectos conjuntos a pesar de la distancia y el tiempo. Don Tapscott alude desde inicios de la década de los noventa a esta nueva generación e identifica con la letra N, por el anglicismo "Net" (red), en referencia a la presencia e influencia de las redes beneficiadas por el uso de la computadora e internet. La Net Gen, que incluye 
a la Generación Y o Millenials (1980-1993) y a la Generación Z (1994-2009), considera unidireccional a la televisión, ya que no permite intervenir en las grillas de programación y contenidos (Ferreiro R., 2000).

Las TIC son la punta de iceberg de un fenómeno que influye y constituye conjuntamente con otras causas el contexto socio-cultural en que se desenvuelven los miembros de esta generación. Se tiende a considerar a la actual generación como un grupo muy heterogéneo de niños, adolescentes y jóvenes, en contacto directo y casi permanente con las TIC, lo que provoca una influencia muy trascendente en su vida (Ferreiro R., 2000).

Esta generación se muestra abierta al cambio, a consumir nuevas y flamantes tecnologías, y también a nuevas conductas y relaciones sociales, a modos de apreciar la vida desde otro ángulo, con o sin nuevos prejuicios morales. Los Nets ostentan una pasmosa capacidad de adaptación a toda actividad que involucra el empleo de las TIC en general y en particular de computadora e internet. Los miembros de esta generación son eminentemente activos, visuales, propensos al intercambio y emprendedores mediante el empleo de las TIC, dada la naturaleza integrativa, visual, auditiva y kinestésica de las TIC en general, y de la computadora e internet en particular y de sus múltiples aplicaciones (Ferreiro R., 2000). La revolución tecnológica en las sociedades estimula también cambios en las maneras en que los jóvenes practican las formas de consumo y producción cultural, las maneras de relacionarse, de comunicarse, de informarse y de aprender, así como muestran diversos estudios y análisis de tipo sociológico, antropológico, comunicacional y educativo (Turkle, 1995; Postman, 1991; Lankshear y Knobel, 2008). Las expresiones tecnológicas primordiales a que se hace referencia actualmente, son aquellas relacionadas con la telefonía móvil (chats en whatsapp, grupos de conversación, sms), los videojuegos (tanto los que se juegan off-line como on-line) y todas aquellas intervenidas por Internet, tanto en su versión web 1.0 (correo electrónico, chat, foros electrónicos, buscadores de información, webs temáticas) como en la versión web 2.0 (blogs, fotologs, wikis, mensajería instantánea con video y audio, podcast, redes sociales) (Escofet Roig et al, 2011).

La generación Net puede pasar horas en las computadoras sin notar del paso del tiempo y, en consecuencia, olvidarse de tareas a cumplir y compromisos contraídos. Esto puede alterar su horario de vida, afectando las horas de sueño, imprescindible para el recupero de la capacidad funcional del sistema neuro-físico, provocando estrés, ansiedad, depresión, fobias, o bien cuadros psicológicos agudos que afectan su salud física. En contraste con las virtudes antes detalladas, los Net Gen son ambivalentes, y a la vez que quieren ser tomados en cuenta para la resolución de problemas y toma de decisiones, demuestran apego a un mundo de fantasía creado por los medios de comunicación masivos, el cine e Internet. Es evidente que, por ejemplo, son biológicamente muy maduros y psicológicamente dependientes de las personas que les rodean. Exhiben a veces un conjunto de actitudes infantiles, desde caprichos hasta el rechazo a la responsabilidad, pese a su edad y nivel académico (Ferreiro R., 2000).

Mientras que la generación Baby Boomer tenía un sólo canal de televisión, tal vez una o dos marcas de gaseosas y un estreno cinematográfico semanal, por dar un ejemplo, la Net Gen accede a un sinfín de opciones y elecciones al alcance de la mano. En comparación con los riesgos que confrontaba la generación $\mathrm{X}$, que le precedió en el tiempo y relacionados con el consumo de tabaco, alcohol y drogas, los Net Gen tienen que lidiar con el 
aumento y variedad de la pornografía, la pornografía cibernética, y el empleo abundante, impredecible, y mal intencionado de infinidad de portales electrónicos diseñados ex profeso para tentarlos con todo ello (Ferreiro R., 2000).

Por efecto de ello y de múltiples causas es posible que el desarrollo humano se haya acelerado. Esta velocidad en el desarrollo humano estaría causada por múltiples factores, algunos socioeconómicos, otros ambientales, un tercer grupo relacionado con causas alimentarias, además de los procesos migratorios y el intercambio genético entre los miembros de una pareja (Ferreiro R., 2000).

A partir de la segunda década del nuevo milenio asistimos al surgimiento de la Generación Touch. Esta corresponde a personas nacidas en los años 2010 a 2020; se caracteriza por interactuar con los medios digitales directamente con las manos: tocar lo virtual es la manera habitual en que los Touch interactúan con los dispositivos. Esta generación está en pleno desarrollo, los miembros mayores tienen entre 7 y 8 años, y representa uno de los mayores desafíos educativos de la historia (Kostyrka-Allchorne K, et al., 2017).

En oposición con la perspectiva positiva de los posibles efectos del uso intensivo y generalizado de las TIC, tanto en las actividades de la vida cotidiana como en el aprendizaje, algunos autores se han referido también a los inconvenientes y contingencias de un papel dominante de las tecnologías como intermediarias de las prácticas socioeducativas entre los más jóvenes. Monereo (2004) delinea este lado oscuro de lo que él llama la construcción virtual de la mente: relativismo y confusión cognitiva, habilidades interpersonales deficitarias, mengua del compromiso social, infoxicación o intoxicación con información, tecno autismo o aislamiento social promovido por el abuso de la tecnología, son sólo algunos de los posibles problemas aludidos (Ferreiro R., 2000).

\section{¿Qué retos significativos presenta la Generación Net para la didáctica del SXXI?}

Desde una perspectiva educativa, llama la atención de los analistas de esta generación la inmediatez de sus acciones y toma de decisiones. Los miembros de la Net Gen se caracterizan por procesos de atención con márgenes amplios. En forma simultánea a la tarea que realizan, escuchan música, mantienen y trabajan con varias ventanas en paralelo, atienden el teléfono, chatean por whatsapp, suben fotos a Instagram, responden a una pregunta que se les hace en ese momento, entre otras. O sea, ostentan una capacidad de atención diversificada y distinta de la de generaciones anteriores, caracterizadas por profundizar más que por abarcar contenidos variados. Lo descripto posee un peculiar alcance pedagógico: es casi imposible mantenerlos atentos en un aula de clase tradicional, con un docente sentado exponiendo un contenido que muchas veces ellos mismos pueden consultar directamente en internet, mientras que a su vez intercambian con otros, localizan otras fuentes, pueden elaborar mapas o visualizaciones, es decir, y acá radica la clave del cambio generacional: participan de manera activa en la construcción del conocimiento (Ferreiro R., 2000).

El conocimiento declarativo (conceptos, leyes, modelos teóricos) y una serie de habilidades intelectuales se prestan para el trabajo “en línea”, mientras que el trabajo presencial en 
clase, se debiera aprovechar de forma didáctica para el desarrollo del contenido procesal (habilidades psico-motoras, sociales, actitudes y valores). El uso de computadora e internet favorece la actividad independiente (observación, exploración, búsqueda, comparación, ordenamiento, clasificación, toma de decisiones, procesamiento de la información y una serie de operaciones mentales: análisis y síntesis, abstracción y generalización) y también la actividad con otros en tiempo real (sincrónica) y en tiempo diferido (asincrónica), lo que facilita el trabajo en equipo cooperativo y las relaciones sociales con otros para aprender (Ferreiro R., 2000).

Esto lleva a reflexionar si las instituciones de educación superior y su plantel docente están preparados para responder a las expectativas de estos estudiantes y, a continuación, habría que preguntarse si en parte los problemas educativos relacionados con la deserción estudiantil tienen que ver con este desfasaje entre la naturaleza de los alumnos y los sistemas de formación actuales. Pareciera que, a partir de estos aportes, los estudiantes universitarios de hoy debieran ser altamente hábiles en el uso creativo de las tecnologías, en el procesamiento rápido y no lineal de la información, en la interpretación y el manejo del lenguaje audiovisual, en la interacción social en entornos virtuales, entre otros (Ferreiro R., 2000). El empleo de las nuevas tecnologías constituye un reto a vencer más en lo referido a lo pedagógico, que a lo tecnológico propiamente dicho. No se trata de incorporar lo nuevo en lo viejo, o seguir haciendo lo mismo con las tecnologías de punta. Introducir la tecnología por la tecnología misma en la educación sería ilógico, y tarde o temprano resultaría improcedente. No es posible educar a la generación Net sin el uso de las tecnologías que los unen y marcan como generación, en un nivel de necesidades de aprendizaje formal. Las investigaciones demuestran que la Net Gen no aprende de modo lineal, y evidencia los variados y válidos estilos y ritmos de aprendizajes que poseen los miembros de esta generación. La atención desarrolladora a los distintos modos de aprender, debido a los diferentes estilos, ritmos y talentos, y con ello la atención a una diversidad, nunca antes fue puesta en evidencia, ni tomada en consideración (Ferreiro R., 2000).

Todo lo anterior exige del enseñante y educador el desarrollo de toda una serie de competencias profesionales, pedagógicas y didácticas, posiblemente muy dificultosas de poner en práctica en el contexto de universidad masiva. Uno de los retos que la Net Gen le presenta a sus docentes es básicamente la atención personalizada que requiere y demanda en contextos sociales de diversidad y el empleo de las TIC de forma extensiva y masiva (Ferreiro R., 2000).

Prensky (2001) utiliza la ya pública denominación de nativos digitales para describir a estos jóvenes. Según el autor, ellos son parlantes nativos de una lengua digital basada en el uso de las computadoras, los videojuegos y el acceso a Internet y los adultos deberíamos concientizar que nos movemos en la sociedad digital pero con acento. Utilizamos la tecnología pero no de la misma forma que la generación actual. De acuerdo con Prensky, cualquier persona nacida a partir de 1980 podría ser considerada un nativo digital, por lo tanto, se trata de los aprendices del nuevo milenio: adolescentes y jóvenes que ocupan distintos niveles del sistema educativo hasta la formación universitaria (Trinder, Guiller, Margaryan, Littlejohn, Nicol, 2008). 


\section{¿Existen diferencias entre los propios estudiantes?}

Si bien hay una gran diversidad de usos entre la población de estudiantes, en especial parece reconocerse en este estudio que las competencias digitales básicas no precisamente se trasladan al uso de las tecnologías con fines de estudio. Lorenzo, Oblinger y Dziuban (2006:4) aseveran que "no todos los estudiantes se benefician de la tecnología. En la educación superior, existe una alta diversidad de estudiantes con capacidades de alfabetización digital muy variadas". La falta de homogeneidad es uno de los principales resultados de esta investigación, que muestra la relación entre el uso de la tecnología y los estudiantes que ingresan a la universidad durante el primer año (Escofet Roig et al, 2011).

Socializar y conectarse con los demás no constituye el único uso de las tecnologías. En el informe de Trinder et al. (2008) se hace saber que un 59\% de los estudiantes ingleses utilizan con frecuencia la web para sus estudios: para buscar información, acceder a las plataformas virtuales institucionales, comunicarse con los compañeros del curso para realizar trabajos, etcétera. Algo que actualmente ya sucede en las aulas y talleres universitarios en Argentina y Latinoamérica. También, se comprueba que, ciertamente en la práctica, algunos profesores universitarios están proporcionando este tipo de canales para desarrollar las experiencias y prácticas formativas (Escofet Roig et al, 2011).

En la mayoría de las investigaciones se muestra cómo estos instrumentos pueden ser utilizados con éxito en la formación. No obstante, los casos que se presentan en las mismas son prácticas aisladas que, en general están promovidas por profesores entusiastas que no obtienen apoyo o compromiso por parte de la institución. Como afirma un informe, frecuentemente el uso de herramientas web 2.0 supone un vínculo efectivo entre un medio formal y uno informal, aportando a los estudiantes nuevas formas de acceder y captar conocimiento, al conectar el contenido del curso con la experiencia de la vida real (AlaMutka et al., 2009).

\section{Algunos conceptos para reflexionar}

Si algo está claro es que este tipo de temática está lejos de cerrarse o que se arribe a conclusiones definitivas. La Net Gen es una generación diferente, como todas la han sido en su momento, pero con sorprendentes y variadas perspectivas por el momento histórico que le toca vivir. Compone el porcentaje mayoritario en términos estadísticos y demográficos de la sociedad contemporánea. Por caso, sólo en los EEUU son algo más de 88 millones, mucho más de lo que en su época fue la Baby Boomer. Crean a su vez un reto a nivel social general, en los procesos de democratización, comercio y mercado, entretenimiento y diversión, y ciertamente en las instituciones tradicionalmente dedicadas a la formación de las nuevas generaciones (Ferreiro R., 2000).

Concluyendo estas reflexiones y aportes se podría señalar que, para su inserción en un sistema que posibilite su pleno desarrollo, la generación Net requiere el pasaje de:

Aprendizaje lineal a...... nuevos contextos de aprendizaje.

Enseñanza repetidora y memorística a...... construcción social del conocimiento. 
Aprendizaje competitivo a...... aprendizaje cooperativo. Enseñanza individualista a...... interdependencia social.

Docente trasmisor a......docente mediador.

Aprendizaje institucional a.......aprendizaje permanente e ilimitado en tiempo/espacio. Aprendizaje por compromiso u obligación a...... aprendizaje por placer.

Aprendizaje centrado en docente y contenido a......aprendizaje centrado en el desarrollo personal, técnico y profesional del estudiante.

La generación Net no es mejor o peor que otras en su momento. Eso sí, tiene extraordinarias potencialidades y posibilidades dado su momento histórico, y lo que es su principal capital respecto de anteriores generaciones: participan de manera activa en la construcción del conocimiento. De nuestra atención desarrolladora y acompañamiento dependerá en gran medida su correcta inserción y contribución social (Ferreiro R., 2000).

Hay que reconocer que nunca antes como ahora, históricamente se han estudiado y tenido en cuenta las particularidades de las nuevas generaciones con vistas a encarar metodologías de enseñanza. Ello se debe a diversos factores, uno de ellos, son los contundentes rasgos propios y diferencias con generaciones anteriores.

Gestionar de forma inteligente los contrastes entre los miembros de una institución, se refiere a considerar las particularidades generacionales con todo lo que ello implica, haciendo foco en las formas de comunicación, factores que los motivan, y en particular en tener la capacidad de aprovechar esas diferencias como valores agregados, y no como obstáculos, que impulsen el desarrollo de las tareas en común. Implementar estrategias de comunicación efectivas que permitan a las personas conocer los diferentes puntos de vista de cada una de estas y así aspirar a disminuir los prejuicios que se pueden generar sobre las actitudes y comportamientos de los miembros de cada generación. Identificar las diferencias generacionales en base a sus valores, comportamientos, perfil, trabajo e influencias. Es una ardua tarea que compete a las instituciones y enseñantes de hoy.

Vale imaginar que en un futuro próximo los miembros de las generaciones Baby Boomers y $X$, que hoy asumen los roles en docencia y autoridad institucional, serán reemplazados en esos roles por los Nets, hoy aprendices, con todas sus variantes, ...supuestamente estarían resueltas las dificultades de enseñanza-aprendizaje que representan hoy las diferencias generacionales y de paradigmas... no es tan fácil prefigurar cuáles serán las nuevas generaciones de aprendices ni los nuevos paradigmas que moldearán sus estructuras, cuáles tecnologías y avances científicos manejarán o los manejarán a ellos... ni la velocidad de los cambios y contextos sociales....tal vez habrá que dejar estas fantasías para los creadores de ciencia-ficción.

\section{Referencias bibliográficas}

Ala-Mutka, K.; Bacigalupo, M.; Kluzer, S.; Pascu, C.; Punie, Y. y Redecker, C. (2009). Learning 2.0: The impact of Web 2.0. Innovations on education and training in Europe. Institute for Prospective Technological Studies. Joint Research Centre. European Commission. Disponible en: http://ipts.jrc.ec.europa.eu/publications/pub.cfm?id=2139. 
Castells, M. (1997-98). La era de la información (3 vols.). Madrid: Alianza.

Castells, M. (2001). La galaxia Internet. España: Ed. Arete.

Coupland D. (1991). Generation X: Tales for an Accelerated Culture. Nueva York: St. Martin's Press.

Downes, S. (2004). Educational blogging, Educause Review, 39(5). Disponible http://connect. educause.edu/Library/educause+Review/EducationalBlogging/40493

European Commission (2001). Communication: Making a European area of lifelong learning a reality. Disponible en: http://www.europa.eu.int/comm/education/life/index.htm

Ferreiro, R. (2000). Nuevos ambientes de aprendizaje, INPUT. No. 21, Barcelona. pp. 72-74.

Kahnwald, N. (2009). Social software as a tool for informal learning, Elearning Europa, Disponible en: http://www.elearningeuropa.info/files/media/media19951.pdf.

Kostyrka-Allchorne K., Cooper NR, Simpson A. (2017). Touchscreen generation: children's current media use, parental supervision methods and attitudes towards contemporary media. Acta Podiatrica; 106: 654-662.

Lévy, P. (1998). Becoming virtual: reality in the Digital Age. Nueva York: Plenum Trade.

McLuhan, M. (1971). Guerra y paz en la aldea global. Barcelona: Martínez Roca.

Monereo, C. (2004). La construcción virtual de la mente: implicaciones psicoeducativas, Interactive Educational Multimedia, 9. Disponible en: http://www.ub.edu/multimedia/ iem/down/c9/Construction_of_the_mind_\%28SPA\%29.pdf

Negroponte, N. (1995). El mundo digital. Barcelona: Ediciones B.

Negroponte, N. (1996). Ser digital. México: Océano.

Prensky, M. (2001). Digital Natives, digital inmigrants, On the Horizon, 9 (5). Disponible en: http://www.marcprensky.com/writing/Prensky\%20-\%20Digital\%20Natives,\%20 Digital\%20Immigrants\%20-\%20Part1.pdf

Prensky, M. (2009). H. sapiens digital: From digital immigrants and digital natives to digital wisdom, Innovate, 5 (3). Disponible en: http://www.innovateonline.info/pdf/vol5_ issue3/H._Sapiens_Digital___From_Digital_Immigrants_and_Digital_Natives_to_ Digital_Wisdom.pdf

Tapscott, D. (1998). Growing up digital: The rise of the Net generation. Nueva York: McGraw-Hill.

Trinder, K; Guiller, J; Margaryan, A.; Littlejohn, A; Nicol, D. (2008). Learning from digital natives: bridging formal and informal learning. Research project report. The Higher Education Academy. Glasgow Caledonian University. Disponible en: http://www.academy. gcal.ac.uk/ldn/LDNFinalReport.pdf.

3er Encuentro BID de Enseñanza y Diseño, Madrid (2011). Disponible en: http://bid-dimad. org/encuentro/documentos/Memoria_3er-Encuentro-BID.pdf

$5^{\circ}$ Encuentro BID De Centros Iberoamericanos De Enseñanza De Diseño, (2013) Central de Diseño /Matadero Madrid. Disponible en: http://bid-dimad.org/septimoencuentro/ wp-content/uploads/2017/04/firmantes-encuentro.pdf

$6^{\circ}$ Encuentro Bid De Centros Iberoamericanos De Enseñanza De Diseño, (2015), Central de Diseño, Matadero, Madrid Organiza: DIMAD. Disponible en: http://bid-dimad.org/ sextoencuentro/innovacion-docente/

7o Encuentro Bid De Centros Iberoamericanos de Enseñanza de Diseño, (2017), Central de Diseño, Matadero, Madrid Organiza: DIMAD. Disponible en: http://bid-dimad.org/ septimoencuentro/es/disenar-para-un-mundo-complejo-2/ 
Abstract: Teaching-learning design poses challenges in the context of the current global paradigm. The generations of Baby-Boomers (1945-60) and X (1961-79), trained in times of cold war, are the didactic organizers of the NET generations or of digital natives: Millenials (1980-1993), Z (1994- 2009) and Touch (2010-today). Quantitative and qualitative changes occur at an accelerated speed in the economic, political, social, environmental, technological and symbolic dimensions from the end of the 20th century and the beginning of the 21st. New teaching / learning modalities product of generational changes, invite critical reflection.

Keywords: teaching - learning - design - changes - models - generations - critical reflection - context.

Resumo: $O$ design de ensino-aprendizagem apresenta desafios no contexto do atual paradigma global. As gerações de Baby-Boomers (1945-60) e X (1961-79), treinados em tempos de guerra fria, são os organizadores didáticos das gerações NET ou dos nativos digitais: Millenials (1980-1993), Z (1994-2009) e Touch (2010-hoje). As mudanças quantitativos e qualitativos são produzidos a uma velocidade acelerada nas dimensões econômica, política, social, ambiental, tecnológica e simbólica a partir do final do século XX e início do XXI. Novas modalidades de ensino / aprendizagem produto de mudanças geracionais, convidam à reflexão crítica.

Palavras chave: Ensino - aprendizagem - design - mudanças - modelos - gerações - reflexão crítica - contexto.

[Las traducciones de los abstracts fueron supervisadas por el autor de cada artículo] 